\title{
Infantile Gastroesophageal Reflux
}

National Cancer Institute

\section{Source}

National Cancer Institute. Infantile Gastroesophageal Reflux. NCI Thesaurus. Code

C113396.

Effortless regurgitation of gastric contents that commonly occurs in infants, usually right after feeding or burping. 